\title{
GRADED 3-CALABI-YAU ALGEBRAS AS ORE EXTENSIONS OF 2-CALABI-YAU ALGEBRAS
}

\author{
JI-WEI HE, FRED VAN OYSTAEYEN, AND YINHUO ZHANG
}

(Communicated by Birge Huisgen-Zimmermann)

\begin{abstract}
We study a class of graded algebras obtained from Ore extensions of graded Calabi-Yau algebras of dimension 2. It is proved that these algebras are graded Calabi-Yau and graded coherent. The superpotentials associated to these graded Calabi-Yau algebras are also constructed.
\end{abstract}

\section{INTRODUCTION}

Recently, Smith studied in $\mathrm{Sm} 2$ a remarkable graded Calabi-Yau algebra $B$ of dimension 3 constructed from the octonions. Amongst other things, Smith proved that $B$ is a graded Ore extension of an Artin-Schelter regular algebra of global dimension 2 and uses that fact to show that $B$ is graded 3-Calabi-Yau and graded coherent.

In this note, we show that the Calabi-Yau property and the coherence of $B$ do not occur incidentally. A large class of graded algebras that are Ore extensions of graded Calabi-Yau algebras are themselves graded Calabi-Yau. The main result of this note is the following.

Theorem 0.1. Let $V$ be a finite dimension vector space with basis $\left\{x_{1}, \ldots, x_{n}\right\}$, let $M$ be an invertible $n \times n$ anti-symmetric matrix, and define

$$
r=\left(x_{1}, \ldots, x_{n}\right) M\left(\begin{array}{c}
x_{1} \\
\vdots \\
x_{n}
\end{array}\right) \in T(V) .
$$

Let $A=T(V) /\langle r\rangle$, where $\langle r\rangle$ is the ideal of $T(V)$ generated by $r$. Let $\delta$ be a degree-one graded derivation of $T(V)$ such that $\delta(r)=0$. Then $\delta$ induces a graded derivation $\bar{\delta}$ on $A$. Let $B=A[z ; \bar{\delta}]$ be the Ore extension of $A$ defined by $\bar{\delta}$. Then the following hold:

(i) B is a graded 3-Calabi-Yau algebra.

(ii) Let $\widehat{V}=V \oplus \mathbb{k} z$, and $Q=\left(\begin{array}{cc}-1 & 0 \\ 0 & M\end{array}\right)$. Let $w=\left(z, x_{1}, \ldots, x_{n}\right) Q\left(\begin{array}{c}r \\ r_{1} \\ \vdots \\ r_{n}\end{array}\right)$, where $r_{i}=z \otimes x_{i}-x_{i} \otimes z-\delta\left(x_{i}\right) \in \widehat{V} \otimes \widehat{V}$ for all $i=1, \ldots, n$. Then

Received by the editors March 16, 2012 and, in revised form, February 17, 2013 and August $13,2013$.

2010 Mathematics Subject Classification. Primary 16S37, 16S38, 16 E65.

Key words and phrases. Calabi-Yau algebra, Ore extension, superpotential. 
$(\alpha \otimes 1 \otimes 1)(w)=(1 \otimes 1 \otimes \alpha)(w)$ for all $\alpha \in(\widehat{V})^{*}$, and $A[z ; \bar{\delta}] \cong T(\widehat{V}) /\left\langle\partial_{x_{i}}(w):\right.$ $i=0, \ldots, n\rangle$, where we set $x_{0}=z$ and $\partial_{x_{i}}(w)$ is the cyclic partial derivative of $w$ with respect to $x_{i}$.

(iii) Write $\delta\left(x_{i}\right)=\sum_{s, t=1}^{n} k_{s t}^{i} x_{i} \otimes x_{j}$ for all $i=1, \ldots, n$. Assume there is an integer $j$ such that $k_{j j}^{i}=0$ for all $i=1, \ldots, n$, and $M$ is a standard anti-symmetric matrix. Then $B$ is graded coherent.

Most of this note is devoted to the proof of Theorem 0.1 However, we will go a bit further to discuss the properties of the algebra $B$. Smith's algebra in [Sm2 is an example satisfying the conditions in the theorem. We will provide a few more examples. We remark that any quadratic algebra $A$ defined by an invertible anti-symmetric matrix as in the above theorem is isomorphic to a quadratic algebra defined by a standard anti-symmetric matrix (see Convention 2.3 for the definition), because, for every invertible anti-symmetric matrix $M$, there is an invertible matrix $P$ such that $P^{t} M P$ is a standard anti-symmetric matrix.

Remark 0.2 . Let $V$ be a finite dimensional vector space with basis $\left\{x_{1}, \ldots, x_{n}\right\}$. Take an element $r \in V \otimes V$. Since $V \otimes V \cong \operatorname{Hom}_{\mathbb{k}}\left(V^{*}, V\right)$, the element $r$ corresponds to a linear map $f_{r}: V^{*} \rightarrow V$. The rank of $r$, denoted by $\operatorname{rank}(r)$, is defined to be the rank of $f_{r}$ (cf. [Z2, Introduction]). One sees

$$
\operatorname{rank}(r)=\min \left\{m \mid r=u_{1} \otimes v_{1}+\cdots+u_{m} \otimes v_{m}, \text { for some } u_{i}, v_{i} \in V\right\} .
$$

It has been shown that certain features of the algebra $T(V) /\langle r\rangle$ entirely depend on $\operatorname{rank}(r)$ (cf. [Z2, Theorem 0.1]). If $M$ is an $n \times n$ matrix and

$$
r=\left(x_{1}, \ldots, x_{n}\right) M\left(\begin{array}{c}
x_{1} \\
\vdots \\
x_{n}
\end{array}\right) \in V \otimes V,
$$

then $\operatorname{rank}(r)=\operatorname{rank}(M)$. Therefore, the condition that $M$ is invertible in Theorem 0.1 is equivalent to the condition that $\operatorname{rank}(r)=n$.

Throughout $\mathbb{k}$ is a fixed field. The unadorned $\otimes$ means $\otimes_{\mathbb{k}}$. Let $U=\oplus_{n \in \mathbb{Z}} U_{n}$ be a graded vector space, and $l$ an integer. We write $U(l)$ for the graded vector space with degree $k$ component $U(l)_{k}=U_{k+l}$.

A connected graded algebra $A$ is called a graded Calabi-Yau algebra of dimension $d$, or simply graded $d$-CY algebra (cf. Gin]), if

(i) $A$ is homologically smooth; that is, $A$ has a finite resolution by finitely generated graded projective left $A^{e}$-modules, where $A^{e}=A \otimes A^{o p}$ is the enveloping algebra of $A$;

(ii) the projective dimension of $A$ as a left $A^{e}$-module is $d$, and $\operatorname{Ext}_{A^{e}}^{i}(A, A \otimes A)=0$ if $i \neq d$ and $\operatorname{Ext}_{A^{e}}^{d}(A, A \otimes A) \cong A(l)$ for some integer $l$ as a right $A^{e}$-module.

We refer to [Z1] (also, cf. [Be] and [DV]) for the basic properties of a graded 2-CY algebra.

\section{Ore extensions of graded Calabi-Yau algebras of Dimension 2}

Let $V$ be a vector space with basis $\left\{x_{1}, \ldots, x_{n}\right\}$. Let $A$ be a graded quotient algebra of $T(V)$. If $A$ is a graded 2-CY algebra, then it is defined by an $n \times n$ invertible anti-symmetric matrix $M$ [Z1] (also, cf. [Be, Proposition 3.4]); that is, 
$A \cong T(V) /\langle r\rangle$ with $r=\left(x_{1}, \ldots, x_{n}\right) M\left(x_{1}, \ldots, x_{n}\right)^{t}$. Henceforth, $A=T(V) /\langle r\rangle$ with $r=\left(x_{1}, \ldots, x_{n}\right) M\left(x_{1}, \ldots, x_{n}\right)^{t}$ for some fixed invertible anti-symmetric ma$\operatorname{trix} M$. Let $\pi: T(V) \rightarrow A$ be the natural projection map. Since degree $(r)=2$, we can, and we will, identify $V$ with $A_{1}$ through the projection $\pi$.

Let $\delta: V \rightarrow V \otimes V$ be a linear map. Then $\delta$ extends in a unique way to a degree-one derivation (also denoted by $\delta$ ) of $T(V)$. If $\delta(r) \in\langle r\rangle$, then $\delta$ induces a derivation $\bar{\delta}$ on $A$.

From now on, we assume that $\delta(r) \in\langle r\rangle$. Let $B=A[z ; \bar{\delta}]$ be the graded Ore extension of $A$ by the derivation $\bar{\delta}$; that is, we view $z$ as an element of degree one, and $z a=a z+\bar{\delta}(a)$ for all $a \in A$.

Zhang proved in [Z1 that $A$ is a Koszul algebra of global dimension 2, and the minimal projective resolution of ${ }_{A} \mathbb{k}$ can be written as follows:

$$
0 \longrightarrow A \otimes \mathbb{k} r \stackrel{\bar{d}^{-2}}{\longrightarrow} A \otimes V \stackrel{\bar{d}^{-1}}{\longrightarrow} A \stackrel{\varepsilon}{\longrightarrow} A \mathbb{k} \longrightarrow 0
$$

where $\varepsilon$ is the augmentation map, $\bar{d}^{-1}(1 \otimes x)=\pi(x)$ for all $x \in V$, and $\bar{d}^{-2}(1 \otimes r)=$ $r \in A_{1} \otimes V$. Since $B$ is an Ore extension of $A, B$ is a Koszul algebra of global dimension 3. Note that $B_{A}$ is a free $A$-module. Applying $B \otimes_{A}-$ to the sequence (11), we obtain the exact sequence

$$
0 \longrightarrow B \otimes \mathbb{k} r \stackrel{d^{-2}}{\longrightarrow} B \otimes V \stackrel{d^{-1}}{\longrightarrow} B \longrightarrow B / B A_{\geq 1} \longrightarrow 0
$$

where the unlabeled map is the natural projection map, $d^{-1}(1 \otimes x)=\pi(x) \in B_{1}$ for all $x \in V$, and $d^{-2}(1 \otimes r)=r \in B_{1} \otimes V$.

Lemma 1.1. Suppose that $\delta(r)=0$ and let $B=A[z ; \bar{\delta}]$ be as above. We have the following morphism of cochain complexes:

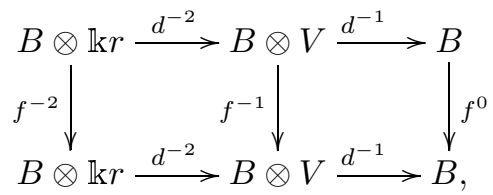

where the vertical arrows are left $B$-module morphisms $f^{-2}(1 \otimes r)=z \otimes r$, $f^{-1}(1 \otimes x)=z \otimes x-\delta(x)$ for all $x \in V$, and $f^{0}(1)=z$.

Proof. We write $r=\sum_{i=1}^{n} u_{i} \otimes x_{i}$ with all $u_{i} \in V$, and assume $\delta\left(x_{i}\right)=\sum_{j=1}^{n} y_{i j} \otimes x_{j}$ for all $i=1, \ldots, n$ with all $y_{i j} \in V$. We prove the commutativity of the left square. The commutativity of the right one is easy. The identity $\delta(r)=0$ is equivalent to $\sum_{i=1}^{n} \delta\left(u_{i}\right) \otimes x_{i}+\sum_{i=1}^{n} u_{i} \otimes \delta\left(x_{i}\right)=0$, which is in turn equivalent to $\sum_{i=1}^{n} \delta\left(u_{i}\right) \otimes x_{i}+\sum_{i=1}^{n} \sum_{j=1}^{n} u_{i} \otimes y_{i j} \otimes x_{j}=0$. Applying the map $\pi \otimes 1: T(V) \otimes V \rightarrow$ $A \otimes V$ to the last identity, we obtain $\sum_{i=1}^{n} \bar{\delta}\left(u_{i}\right) \otimes x_{i}+\sum_{i=1}^{n} \sum_{j=1}^{n} u_{i} y_{i j} \otimes x_{j}=0$. Hence

$$
\bar{\delta}\left(u_{i}\right)=-\sum_{j=1}^{n} u_{j} y_{j i}
$$


for all $i=1, \ldots, n$. The following equations hold:

$$
\begin{aligned}
f^{-1} \circ d^{-2}(1 \otimes r) & =f^{-1}\left(\sum_{i=1}^{n} u_{i} \otimes x_{i}\right) \\
& =\sum_{i=1}^{n} u_{i} z \otimes x_{i}-\sum_{i=1}^{n} \sum_{j=1}^{n} u_{i} y_{i j} \otimes x_{j} \\
& =\sum_{i=1}^{n}\left(u_{i} z-\sum_{j=1}^{n} u_{j} y_{j i}\right) \otimes x_{i}
\end{aligned}
$$

and

$$
\begin{aligned}
d^{-2} \circ f^{-2}(1 \otimes r) & =d^{-2}(z \otimes r)=\sum_{i=1}^{n} z u_{i} \otimes x_{i} \\
& =\sum_{i=1}^{n}\left(u_{i} z+\bar{\delta}\left(u_{i}\right)\right) \otimes x_{i} .
\end{aligned}
$$

By equation (3),$f^{-1} \circ d^{-2}(1 \otimes r)=d^{-2} \circ f^{-2}(1 \otimes r)$. Hence the left square of the diagram commutes.

The mapping cone of the morphism in Lemma 1.1 provides a graded projective resolution of the trivial module ${ }_{B} \mathbb{k}$ (see also, $\left.\mathrm{GS}, \mathrm{Ph}\right]$ ).

Lemma 1.2. Let $r$ and $B$ be the same as in Lemma 1.1. The minimal projective resolution of ${ }_{B} \mathbb{k}$ is as follows:

$$
0 \longrightarrow B \otimes \mathbb{k} r \stackrel{\partial^{-3}}{\longrightarrow} B \otimes \mathbb{k} r \oplus B \otimes V \stackrel{\partial^{-2}}{\longrightarrow} B \otimes V \oplus B \stackrel{\partial^{-1}}{\longrightarrow} B \longrightarrow \mathbb{k} \longrightarrow 0,
$$

where $\partial^{-3}=\left(\begin{array}{c}f^{-2} \\ -d^{-2}\end{array}\right), \partial^{-2}=\left(\begin{array}{cc}d^{-2} & f^{-1} \\ 0 & -d^{-1}\end{array}\right)$, and $\partial^{-1}=\left(d^{-1}, f^{0}\right)$.

Let $A^{!}$be the quadratic dual of $A$. As graded vector spaces $A_{0}^{!} \cong \mathbb{k}, A_{1}^{!} \cong V^{*}$ and $A_{2}^{!} \cong \mathbb{k} r^{*}$, where $r^{*} \in(\mathbb{k} r)^{*}$ defined by $r^{*}(r)=1$. The multiplication on $A^{!}$is given by: $\alpha \beta=\left(a_{1}, \ldots, b_{n}\right) M\left(b_{1}, \ldots, b_{n}\right)^{t} r^{*}$, for $\alpha=a_{1} x_{1}^{*}+\cdots+a_{n} x_{n}^{*}$ and $\beta=b_{1} x_{1}^{*}+\cdots+b_{n} x_{n}^{*}$ in $V^{*}$ (cf. [HVZ2, Section 3]), where $\left\{x_{1}^{*}, \ldots, x_{n}^{*}\right\}$ is the basis of $V^{*}$ dual to the basis $\left\{x_{1}, \ldots, x_{n}\right\}$. Write $E^{i}(B):=\operatorname{Ext}_{B}^{i}\left({ }_{B} \mathbb{k},{ }_{B} \mathbb{k}\right)$ and $E(B):=\bigoplus_{i>0} E^{i}(B)$. Then $E(B)$ is a graded algebra with the degree $i$ component $E^{i}(B)$. The minimal projective resolution of ${ }_{B} \mathbb{k}$ above implies that, as graded vector spaces,

$$
E(B) \cong A^{!} \oplus A^{!}(-1) .
$$

We write an element in $E(B)$ as $(\alpha, \beta)$ for some $\alpha, \beta \in A^{!}$, and demote the Yoneda product on $E(B)$ by $(\alpha, \beta) *\left(\alpha^{\prime}, \beta^{\prime}\right)$.

Proposition 1.3. Assume $\delta(r)=0$. Then $A[z ; \bar{\delta}]$ is a 3-CY algebra.

Proof. By [HVZ1, Proposition 3.3]) in the Koszul case, $B=A[z ; \bar{\delta}]$ is Calabi-Yau if and only if $E(B)$ is a graded symmetric algebra. Recall that a finite dimensional graded algebra $E=\bigoplus_{i>0} E^{i}$ is graded symmetric if there is an integer $d$ and a homogeneous nondegenerate bilinear form $\langle-,-\rangle: E \times E \longrightarrow \mathbb{k}(d)$ such that $\langle\alpha \beta, \gamma\rangle=\langle\alpha, \beta \gamma\rangle$ and $\langle\alpha, \beta\rangle=(-1)^{i j}\langle\beta, \alpha\rangle$ for all homogeneous elements $\alpha \in$ $E^{i}, \beta \in E^{j}$ and $\gamma \in E^{k}$. Since the global dimension of $B$ is 3 and $\operatorname{dim} E^{3}(B)=1$, 
$E(B)$ is graded symmetric if and only if, for all elements $\Phi \in E^{1}(B), \Theta \in E^{2}(B)$, $\Phi * \Theta=\Theta * \Phi$. Let $\Phi=(\alpha, k)$ with $\alpha \in A_{1}^{!}=V^{*}$ and $k \in \mathbb{k}$, and $\Theta=\left(r^{*}, \beta\right)$ with $\beta \in V^{*}$. The element $\Phi$ induces a $B$-module morphism $g: B \otimes V \oplus B \longrightarrow{ }_{B} \mathbb{k}$ by $g(1 \otimes x, 1)=\alpha(x)+k$ for all $x \in V$, and the element $\Theta$ induces a $B$-module morphism $h: B \otimes \mathbb{k} r \oplus B \otimes V \longrightarrow{ }_{B} \mathbb{k}$ by $h(1 \otimes r, 1 \otimes x)=1+\beta(x)$ for all $x \in V$. Consider the following diagram:

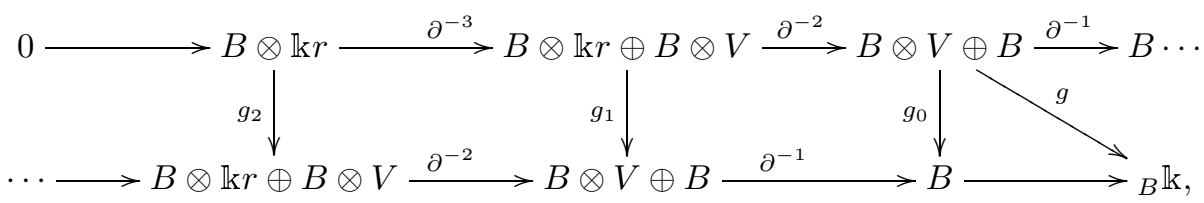

where the vertical arrows are $B$-module morphisms defined as follows. As before, we write $r=\sum_{i=1}^{n} u_{i} \otimes x_{i}$ with all $u_{i} \in V$, and assume $\delta\left(x_{i}\right)=\sum_{j=1}^{n} y_{i j} \otimes x_{j}$ for all $i=1, \ldots, n$ with all $y_{i j} \in V$. Then

$$
\begin{aligned}
g_{0}\left(1 \otimes x_{j}, 1\right) & =\alpha\left(x_{j}\right) 1+k 1 ; \\
g_{1}\left(1 \otimes r, 1 \otimes x_{j}\right) & =\left(\sum_{i=1}^{n} 1 \otimes u_{i} \alpha\left(x_{i}\right)-1 \otimes k x_{j}-\sum_{i=1}^{n} 1 \otimes y_{j i} \alpha\left(x_{j}\right), \alpha\left(x_{j}\right) 1\right) ; \\
g_{2}(1 \otimes r) & =\left(1 \otimes k r, \sum_{i=1}^{n} 1 \otimes u_{i} \alpha\left(x_{i}\right)\right),
\end{aligned}
$$

for all $j=1, \ldots, n$. Since $\delta(r)=0$, it follows that

$$
\sum_{i=1}^{n} \delta\left(u_{i}\right) \otimes x_{i}+\sum_{i=1}^{n} \sum_{j=1}^{n} u_{i} \otimes y_{i j} \otimes x_{j}=0 .
$$

Applying the linear map $1 \otimes 1 \otimes \alpha$ to this identity, one obtains:

$$
\sum_{i=1}^{n} \delta\left(u_{i}\right) \alpha\left(x_{i}\right)+\sum_{i=1}^{n} \sum_{j=1}^{n} u_{i} \otimes y_{i j} \alpha\left(x_{j}\right)=0 .
$$

Using equation (5) and the following computations:

$$
\begin{aligned}
g_{1} \circ \partial^{-3}(1 \otimes r) & =g_{1}(z \otimes r,-r) \\
= & \left(\sum_{i=1}^{n} z \otimes u_{i} \alpha\left(x_{i}\right)+\sum_{i=1}^{n} u_{i} \otimes k x_{i}+\sum_{i=1}^{n} \sum_{j=1}^{n} u_{i} \otimes y_{i j} \alpha\left(x_{j}\right),-\sum_{i=1}^{n} u_{i} \alpha\left(x_{i}\right)\right), \\
\partial^{-2} \circ g_{2}(1 \otimes r) & =\partial^{-2}\left(1 \otimes k r, 1 \otimes \sum_{i=1}^{n} u_{i} \alpha\left(x_{i}\right)\right) \\
& =\left(k r+\sum_{i=1}^{n} z \otimes u_{i} \alpha\left(x_{i}\right)-\sum_{i=1}^{n} \delta\left(u_{i}\right) \alpha\left(x_{i}\right),-\sum_{i=1}^{n} u_{i} \alpha\left(x_{i}\right)\right),
\end{aligned}
$$

we obtain the identity: $g_{1} \circ \partial^{-3}(1 \otimes r)=\partial^{-2} \circ g_{2}(1 \otimes r)$. Hence $g_{1} \circ \partial^{-3}=\partial^{-2} \circ g_{2}$. 
Similar computations show that the second square in the diagram commutes. The commutativity of the triangle in the diagram is obvious. Thus, we have $h \circ g_{2}(1 \otimes r)=$ $h\left(1 \otimes k r, \sum_{i=1}^{n} 1 \otimes u_{i} \alpha\left(x_{i}\right)\right)=k+\sum_{i=1}^{n} \beta\left(u_{i}\right) \alpha\left(x_{i}\right)$. By the definition of the Yoneda product, we have $\Theta * \Phi=\left(r^{*}, \beta\right) *(\alpha, k)=k r^{*}+\beta \alpha$, where $\beta \alpha$ is the product in $A^{!}$. Similarly, we can show that $\Phi * \Theta=k r^{*}-\alpha \beta$. Now $A$ is Calabi-Yau, hence $A^{!}$is graded symmetric; that is, $\alpha \beta=-\beta \alpha$ for all $\alpha, \beta \in A_{1}^{!}$. It follows that $\Phi * \Theta=\Theta * \Phi$. Therefore, $B=A[z ; \bar{\delta}]$ is Calabi-Yau.

The computation in the proof of Proposition 1.3 has given us the formulas of the Yoneda product of $E(B)$.

Corollary 1.4. As vector spaces, $E(B) \cong A^{!} \oplus A^{!}(-1)$. The Yoneda product of $E(B)$ is given as follows: for $\alpha, \beta \in A_{1}^{!}$and $k, k^{\prime} \in \mathbb{k}$,

$$
\left(r^{*}, \beta\right) *(\alpha, k)=(\alpha, k) *\left(r^{*}, \beta\right)=k r^{*}+\beta \alpha
$$

and

$$
\left(\beta, k^{\prime}\right) *(\alpha, k)=\left(\beta \alpha, k^{\prime} \alpha-k \beta-(\beta \otimes \alpha) \circ \delta\right),
$$

where $r^{*}$ is the basis of $A_{2}^{!}$such that $r^{*}(r)=1$.

Proof. The first identity is proved in the proof of Proposition 1.3. Keep the same notions as in the proof of Proposition 1.3. The element $\left(\beta, k^{\prime}\right)$ induces a $B$-module morphism $g^{\prime}: B \otimes V \oplus B \longrightarrow{ }_{B} \mathbb{k}$ by $g^{\prime}(1 \otimes x, 1)=\beta(x)+k^{\prime}$ for all $x \in V$, and $\left(\beta \alpha, k^{\prime} \alpha-k \beta-(\beta \otimes \alpha) \circ \delta\right)$ induces a $B$-module morphism $f: B \otimes \mathbb{k} r \oplus B \otimes V \longrightarrow{ }_{B} \mathbb{k}$ by $f\left(1 \otimes r, 1 \otimes x_{j}\right)=\sum_{i=1}^{n} \beta\left(u_{i}\right) \alpha\left(x_{i}\right)+k^{\prime} \alpha\left(x_{j}\right)-k \beta\left(x_{j}\right)-\sum_{i=1}^{n} \beta\left(y_{j i}\right) \alpha\left(x_{i}\right)$ for all $j=1, \ldots, n$. By the definition of Yoneda product, $\left(\beta, k^{\prime}\right) *(\alpha, k)$ is represented by $g^{\prime} \circ g_{1}$. Now $g^{\prime} \circ g_{1}\left(1 \otimes r, 1 \otimes x_{j}\right)=\sum_{i=1}^{n} \beta\left(u_{i}\right) \alpha\left(x_{i}\right)-k \beta\left(x_{j}\right)-\sum_{i=1}^{n} \beta\left(y_{j i}\right) \alpha\left(x_{i}\right)+$ $k^{\prime} \alpha\left(x_{j}\right)=f\left(1 \otimes r, 1 \otimes x_{j}\right)$ for all $j=1, \ldots, n$. Therefore the second identity follows.

Let $\epsilon: A^{!} \rightarrow A^{!}$be the automorphism of $A^{!}$defined by $\epsilon(\alpha)=-\alpha$ for $\alpha \in A_{1}^{!}$and $\epsilon(\beta)=\beta$ for all $\beta \in A_{2}^{!}$. Let ${ }_{\epsilon} A^{!}$be the graded $A^{!}$-bimodule whose right $A^{!}$-action is the regular action, and whose left $A^{!}$-action is twisted by the automorphism $\epsilon$; that is, for all $\gamma, \theta \in A^{!}$, the left $A^{!}$-action $\gamma \cdot \theta=\epsilon(\gamma) \theta$. Let $I={ }_{\epsilon} A^{!}(-1)$, and let $E\left(A^{!} ; I\right)$ be the trivial extension of $A^{!}$by the $A^{!}$-bimodule $I$. By Corollary 1.4, $E(B)$ is isomorphic to $E\left(A^{!} ; I\right)$.

Corollary 1.5. The Yoneda algebra $E(B)$ is isomorphic to the trivial extension of $A^{!}$by the $A^{!}$-bimodule $I$.

Example 1.6. Consider the Calabi-Yau algebra studied by Smith in Sm2. Let $\mathbb{k}\left\langle x_{1}, \ldots, x_{6}\right\rangle$ be the free algebra generated by six elements. Let $A=\mathbb{k}\left\langle x_{1}, \ldots, x_{6}\right\rangle$ / $\langle r\rangle$, where

$$
r=\left(x_{1}, \ldots, x_{6}\right)\left(\begin{array}{cccccc}
0 & 0 & 0 & 0 & 0 & 1 \\
0 & 0 & 0 & 0 & -1 & 0 \\
0 & 0 & 0 & -1 & 0 & 0 \\
0 & 0 & 1 & 0 & 0 & 0 \\
0 & 1 & 0 & 0 & 0 & 0 \\
-1 & 0 & 0 & 0 & 0 & 0
\end{array}\right)\left(\begin{array}{c}
x_{1} \\
\vdots \\
x_{6}
\end{array}\right)
$$


Define a derivation $\delta: \mathbb{k}\left\langle x_{1}, \ldots, x_{6}\right\rangle \rightarrow \mathbb{k}\left\langle x_{1}, \ldots, x_{6}\right\rangle$ by

$$
\begin{array}{ll}
\delta\left(x_{1}\right)=x_{4} x_{2}-x_{2} x_{4}+x_{3} x_{5}-x_{5} x_{3} & \delta\left(x_{2}\right)=x_{1} x_{4}-x_{4} x_{1}+x_{3} x_{6}-x_{6} x_{3} \\
\delta\left(x_{3}\right)=x_{5} x_{1}-x_{1} x_{5}+x_{6} x_{2}-x_{2} x_{6} & \delta\left(x_{4}\right)=x_{2} x_{1}-x_{1} x_{2}+x_{5} x_{6}-x_{6} x_{5} \\
\delta\left(x_{5}\right)=x_{1} x_{3}-x_{3} x_{1}+x_{6} x_{4}-x_{4} x_{6} & \delta\left(x_{6}\right)=x_{2} x_{3}-x_{3} x_{2}+x_{4} x_{5}-x_{5} x_{4}
\end{array}
$$

Then $\delta(r)=0$, and $B=A[z ; \bar{\delta}]$ is $3-\mathrm{CY}[\mathrm{Sm} 2]$.

Keep the assumption that $\delta(r)=0$. Let $\widehat{V}=V \oplus \mathbb{k} z$. Then $B=A[z ; \bar{\delta}]$ is a quotient algebra of $T(\widehat{V})$. Since $B$ is $3-\mathrm{CY}, B$ is defined by a superpotential [Bo, Theorem 3.1]. Let $\left\{x_{1}^{*}, \ldots, x_{n}^{*}\right\}$ be the basis of $V^{*}$ dual to $\left\{x_{1}, \ldots, x_{n}\right\}$. Recall that a superpotential is an element $w \in \widehat{V} \otimes \widehat{V} \otimes \widehat{V}$ such that $[\alpha w]=[w \alpha]$ for all $\alpha \in(\widehat{V})^{*}$, where $[\alpha w]=(\alpha \otimes 1 \otimes 1)(w)$ and $[w \alpha]=(1 \otimes 1 \otimes \alpha)(w)$. Given a superpotential $w$, the partial derivative of $w$ by $x_{i}$ is defined by $\partial_{x_{i}}(w)=\left[x_{i}^{*} w\right]$ (cf. [BSW]). By [Bo, Theorem 3.1], there is a superpotential $w \in \widehat{V} \otimes \widehat{V} \otimes \widehat{V}$ such that $B \cong T(\widehat{V}) /\left\langle\partial_{x_{i}}(w): i=0, \ldots, x_{n}\right\rangle$ where $x_{0}=z$. We next show that the superpotential $w$ may be written out explicitly. For $i=1, \ldots, n$, let $r_{i}=z \otimes x_{i}-x_{i} \otimes z-\delta\left(x_{i}\right) \in \widehat{V} \otimes \widehat{V}$. Clearly $r, r_{1}, \ldots, r_{n}$ are linearly independent in $\widehat{V} \otimes \widehat{V}$, moreover $B \cong T(\widehat{V}) /\left\langle r, r_{1}, \ldots, r_{n}\right\rangle$. Before we construct the general form of the superpotentials, let us look at the following example.

Example 1.7. Let $\mathbb{k}\langle x, y\rangle$ be the free algebra generated by two elements. Let $\delta: \mathbb{k}\langle x, y\rangle \rightarrow \mathbb{k}\langle x, y\rangle$ be a derivation defined by $\delta(x)=b x^{2}+c y^{2}$ and $\delta(y)=$ $a x^{2}-b x y-b y x$, where $(a, b, c) \in \mathbb{k}^{3}$. Let $r=x y-y x$. Then it is easy to see that $\delta(r)=0$. Therefore, $\delta$ induces a derivation $\bar{\delta}$ on $A=\mathbb{k}[x, y]$. Now $B=A[z ; \bar{\delta}]$ is 3 -CY. A straightforward verification shows that $w=y x z+z y x+$ $x z y-x y z-z x y-y z x-a x^{3}+c y^{3}+b x y x+b x^{2} y+b y x^{2}$ is a superpotential, and $B \cong \mathbb{k}\langle x, y, z\rangle /\left\langle\partial_{x}(w), \partial_{y}(w), \partial_{z}(w)\right\rangle$. Explicitly, the generating relations are $r_{1}=z y-y z-a x^{2}+b y x+b x y, r_{2}=x x-z x+c y^{2}+b x^{2}$ and $r_{3}=y x-x y$.

Proposition 1.8. Assume $\delta(r)=0$. Let $Q=\left(\begin{array}{cc}-1 & 0 \\ 0 & M\end{array}\right)$, and let

$$
w=\left(z, x_{1}, \ldots, x_{n}\right) Q\left(\begin{array}{c}
r \\
r_{1} \\
\vdots \\
r_{n}
\end{array}\right)
$$

where $M$ is an invertible $n \times n$ anti-symmetric matrix, and $r_{i}=z \otimes x_{i}-x_{i} \otimes z-$ $\delta\left(x_{i}\right) \in \widehat{V} \otimes \widehat{V}$ for all $i=1, \ldots, n$. Then

(i) $w$ is a superpotential;

(ii) $A[z ; \bar{\delta}] \cong T(\widehat{V}) /\left\langle\partial_{x_{i}}(w): i=0, \ldots, n\right\rangle$, where we set $x_{0}=z$.

Proof. Let $\left\{m_{i j} \mid i, j=1, \ldots, n\right\}$ be the entries of $M$. Then $r=\sum_{i, j=1}^{n} m_{i j} x_{i} \otimes x_{j}$. Since $\delta(r)=0$, we have $\sum_{i, j=1}^{n} m_{i j} \delta\left(x_{i}\right) \otimes x_{j}=-\sum_{i, j=1}^{n} m_{i j} x_{i} \otimes \delta\left(x_{j}\right)$. Let us 
compute the element $w$.

$$
\begin{aligned}
w= & -z \otimes r+\left(x_{1}, \ldots, x_{n}\right) M\left(\begin{array}{c}
r_{1} \\
\vdots \\
r_{n}
\end{array}\right) \\
= & -\sum_{i, j=1}^{n} m_{i j} z \otimes x_{i} \otimes x_{j}+\sum_{i, j=1}^{n} m_{i j} x_{i} \otimes r_{j} \\
= & -\sum_{i, j=1}^{n} m_{i j} z \otimes x_{i} \otimes x_{j}+\sum_{i, j=1}^{n} m_{i j} x_{i} \otimes z \otimes x_{j} \\
& -\sum_{i, j=1}^{n} m_{i j} x_{i} \otimes x_{j} \otimes z-\sum_{i, j=1}^{n} m_{i j} x_{i} \otimes \delta\left(x_{j}\right) \\
= & -\sum_{i, j=1}^{n} m_{i j}\left(z \otimes x_{i}-x_{i} \otimes z\right) \otimes x_{j}-\sum_{i, j=1}^{n} m_{i j} x_{i} \otimes x_{j} \otimes z+\sum_{i, j=1}^{n} m_{i j} \delta\left(x_{i}\right) \otimes x_{j} \\
= & -\sum_{i, j=1}^{n} m_{i j}\left(z \otimes x_{i}-x_{i} \otimes z-\delta\left(x_{i}\right)\right) \otimes x_{j}-\sum_{i, j=1}^{n} m_{i j} x_{i} \otimes x_{j} \otimes z \\
= & -r \otimes z+\left(r_{1}, \ldots, r_{n}\right) M^{t}\left(\begin{array}{c}
x_{1} \\
\vdots \\
x_{n}
\end{array}\right) .
\end{aligned}
$$

Now it is clear that $\left[x_{i}^{*} w\right]=\left[w x_{i}^{*}\right]$, and $\partial_{x_{i}}(w)=r_{i}$ for all $i=0,1, \ldots, n$, where $r_{0}=r$.

\section{Coherence of $A[z ; \bar{\delta}]$}

Notation and notions are as in the previous section. By [Z1, Theorem 0.2], $A$ is Noetherian if and only if $\operatorname{dim}(V)=2$. Since $B$ is an Ore extension of $A$ in variable $z, B / B z$ is isomorphic to $A$ as a graded left $B$-module. Since $A$ is not left Noetherian when $\operatorname{dim}(V)>2$, neither is $B$. Similarly, $B$ is not right Noetherian when $\operatorname{dim}(V)>2$. Summarizing the foregoing argument, we obtain the following property.

Lemma 2.1. $B=A[z ; \bar{\delta}]$ is Noetherian if and only if $\operatorname{dim}(V)=2$.

Piontkovski showed in [Pi, Theorem 4.1] that any connected graded algebra with a single quadratic relation is graded coherent. Hence $A$ is a graded coherent algebra. So, it is natural to ask whether $B$ is a graded coherent algebra. The answer is affirmative. However, the proof of this property is not trivial because an Ore extension of a coherent ring need not be coherent. In fact, there is a commutative coherent ring $R$ such that the polynomial extension $R[z]$ is not coherent [So. Some other results about the coherence of polynomial rings may be found in $\mathrm{GV}$ ]. Let us recall the definition of a graded coherent algebra.

A graded algebra $D$ is called a graded left coherent algebra if one of the following equivalent conditions is satisfied:

(i) every finitely generated graded left ideal of $D$ is finitely presented; that is, if $I$ is a graded left ideal of $D$, then there is a finitely graded free $D$-module 
$F$ and a surjective morphism $g: F \rightarrow I$ of graded modules such that ker $g$ is also a finitely generated $D$-module;

(ii) every finitely generated graded submodule of a finitely presented graded module is finitely presented;

(iii) the category of all finitely presented graded left $D$-modules is an abelian category.

Similarly we can define a graded right coherent algebra. If a graded algebra is both graded left and right coherent, then it is called a graded coherent algebra.

Let $W=\bigoplus_{i>0} W_{i}$ be a graded vector space with $\operatorname{dim}\left(W_{i}\right)<\infty$ for all $i$. Recall that the Hilbert series of $W$ is defined to be the power series $H_{W}(t)=$ $\sum_{i \geq 0} \operatorname{dim}\left(W_{i}\right) t^{i}$.

Lemma 2.2. Let $V$ be a vector space of dimension $n \geq 4$ with basis $\left\{x_{1}, \ldots, x_{n}\right\}$, and let

$$
M=\left(\begin{array}{cccccc}
0 & \cdots & 0 & 0 & \cdots & 1 \\
\vdots & & \vdots & \vdots & & \vdots \\
0 & \cdots & 0 & 1 & \cdots & 0 \\
0 & \cdots & -1 & 0 & \cdots & 0 \\
\vdots & & \vdots & \vdots & & \vdots \\
-1 & \cdots & 0 & 0 & \cdots & 0
\end{array}\right)
$$

be the invertible $n \times n$ anti-symmetric matrix with entries in the anti-diagonal line 1 or -1 and others 0 . Let $r=\left(x_{1}, \ldots, x_{n}\right) M\left(x_{1}, \ldots, x_{n}\right)^{t}$, and $A=T(V) /\langle r\rangle$. Let $\delta$ be a derivation on $T(V)$ of degree one. We write $\delta\left(x_{i}\right)=\sum_{s, t=1}^{n} k_{s t}^{i} x_{s} \otimes x_{t}$ for all $i=1, \ldots, n$. Assume that $k_{n n}^{i}=0$ for all $i=1, \ldots, n$ and $\delta(r)=0$. Let $\bar{\delta}$ be the derivation on $A$ induced by $\delta$. Write $B=A[z ; \bar{\delta}]$. Then the following hold:

(i) Let $I$ be the ideal of $B$ generated by the elements $x_{1}, \ldots, x_{n-1}$. Then $B / I \cong$ $\mathbb{k}[X, Z]$, where $\mathbb{k}[X, Z]$ is the commutative polynomial algebra in variables $X$ and $Z$;

(ii) Let $L=\mathbb{k} x_{1} \oplus \cdots \oplus \mathbb{k} x_{n-1}$ and $L^{\prime}=\mathbb{k} x_{2} \oplus \cdots \oplus \mathbb{k} x_{n-1}$. Then, as left $B$-modules, $I \cong B \otimes\left(L \oplus L^{\prime} x_{n} \oplus L^{\prime} x_{n}^{2} \oplus \cdots\right)$, where $L^{\prime} x_{n}^{k}(k \geq 1)$ is the vector space spanned by the elements $x_{2} x_{n}^{k}, \ldots, x_{n-1} x_{n}^{k}$.

Convention 2.3. We call an $n \times n(n \geq 2)$ invertible anti-symmetric matrix of the form (6) a standard anti-symmetric matrix. If $M$ is an invertible anti-symmetric matrix, there is an invertible matrix $P$ such that $P^{t} M P$ is standard.

Proof of Lemma 2.2. (i) By assumption, $\delta\left(x_{n}\right)=\sum_{s, t=1}^{n} k_{s t}^{n} x_{s} \otimes x_{t}$ and $k_{n n}^{n}=$ 0 . Therefore $\bar{\delta}\left(x_{n}\right) \in I$ and $B / I$ is a commutative algebra. There is an algebra morphism $g: k[X, Z] \longrightarrow B / I$ defined by $g(X)=x_{n}$ and $g(Z)=z$. Next, we want to construct an algebra morphism from $B / I$ to $\mathbb{k}[X, Z]$. As before, write $\widehat{V}=V \oplus \mathbb{k} z$. First, we define $f: T(\widehat{V}) \longrightarrow \mathbb{k}[X, Z]$ by letting $f\left(x_{i}\right)=0$ for all $i=1, \ldots, n-1, f\left(x_{n}\right)=X$ and $f(z)=Z$. Denote by $\left\langle x_{1}, \ldots, x_{n-1}\right\rangle$ and by $\left\langle z \otimes x_{n}-x_{n} \otimes z\right\rangle$ the ideals of $T(\widehat{V})$ respectively generated by $x_{1}, \ldots, x_{n-1}$ and by $z \otimes x_{n}-x_{n} \otimes z$. Obviously, $\left\langle x_{1}, \ldots, x_{n-1}\right\rangle+\left\langle z \otimes x_{n}-x_{n} \otimes z\right\rangle \subseteq \operatorname{ker} f$. Recall that $B$ is a Koszul algebra and $B=T(\widehat{V}) / J$ where $J=\left\langle r, z \otimes x_{1}-x_{1} \otimes z-\delta\left(x_{1}\right), \ldots, z \otimes\right.$ $\left.x_{n}-x_{n} \otimes z-\delta\left(x_{n}\right)\right\rangle$. Since $\delta\left(x_{i}\right)=\sum_{s, t=1}^{n} k_{s t}^{i} x_{s} \otimes x_{t}$ such that $k_{n n}^{i}=0$ for all $i=1, \ldots, n$, it follows that $\delta\left(x_{i}\right) \in\left\langle x_{1}, \ldots, x_{n-1}\right\rangle$ for all $i=1, \ldots, n$. Hence 
$r, z \otimes x_{1}-x_{1} \otimes z-\delta\left(x_{1}\right), \ldots, z \otimes x_{n-1}-x_{n-1} \otimes z-\delta\left(x_{n-1}\right) \in\left\langle x_{1}, \ldots, x_{n-1}\right\rangle \subseteq \operatorname{ker} f$. Now $z \otimes x_{n}-x_{n} \otimes z-\delta\left(x_{n}\right) \in\left\langle z \otimes x_{n}-x_{n} \otimes z\right\rangle+\left\langle x_{1}, \ldots, x_{n-1}\right\rangle \subseteq$ ker $f$. Hence $J \subseteq$ ker $f$. Therefore, $f$ induces an algebra morphism $\bar{f}: B \longrightarrow \mathbb{k}[X, Z]$. Obviously, $\operatorname{ker} \bar{f} \supseteq I$. Hence $\bar{f}$ in turn induces an algebra morphism $\hat{f}: B / I \longrightarrow \mathbb{k}[X, Z]$. Now it is easy to see that $\hat{f} \circ g=i d=g \circ \hat{f}$. The statement (i) follows.

(ii) Here we make use of the technique from [Sm2, Proposition 7.3]. Let $\mu$ : $B \otimes B \rightarrow B$ be the multiplication of $B$. Then the restriction of $\mu$ defines a left $B$-module morphism (also denoted by $\mu$ ):

$$
\mu: B \otimes\left(L \oplus L^{\prime} x_{n} \oplus L^{\prime} x_{n}^{2} \oplus \cdots\right) \longrightarrow I .
$$

We claim that $\mu$ is surjective. In fact, if we can show that the image $I^{\prime}=\operatorname{im}(\mu)$ is also an ideal of $B$, then $I=I^{\prime}$. So, it suffices to show that $I^{\prime} x_{n} \subseteq I^{\prime}$ and $I^{\prime} z \subseteq I^{\prime}$. Following the generating relation of $A$, we have $x_{1} x_{n}=x_{n} x_{1}+\left(x_{2} x_{n-1}-\right.$ $\left.x_{n-1} x_{2}\right)+\cdots+\left(x_{\frac{n}{2}} x_{\frac{n}{2}+1}-x_{\frac{n}{2}+1} x_{\frac{n}{2}}\right) \in B L \subseteq I^{\prime}$. Therefore $I^{\prime} x_{n} \subseteq I^{\prime}$. In particular, $\bar{\delta}\left(x_{i}\right) \in I^{\prime}$ for all $i=1, \ldots, n$. On the other hand, since $x_{i} z=z x_{i}-\bar{\delta}\left(x_{i}\right)$, it follows that $x_{i} z \in I^{\prime}$ for all $i=1, \ldots, n-1$. For $2 \leq i \leq n-1$, we have $x_{i} x_{n} z=$ $x_{i}\left(z x_{n}-\bar{\delta}\left(x_{n}\right)\right)=x_{i} z x_{n}-x_{i} \bar{\delta}\left(x_{n}\right) \in I^{\prime} x_{n}+x_{i} I^{\prime} \subseteq I^{\prime}$. Now assume $x_{i} x_{n}^{j} z \in I^{\prime}$ for all $j<k$ and $2 \leq i \leq n-1$. Then

$x_{i} x_{n}^{k} z=x_{i} x_{n}^{k-1}\left(z x_{n}-\bar{\delta}\left(x_{n}\right)\right)=\left(x_{i} x_{n}^{k-1} z\right) x_{n}-x_{i} x_{n}^{k-1} \bar{\delta}\left(x_{n}\right) \in I^{\prime} x_{n}+x_{i} x_{n}^{k-1} I^{\prime} \subseteq I^{\prime}$.

Hence $I^{\prime} z \subseteq I^{\prime}$. The claim follows. To show that $\mu$ is injective, we only need to compare the Hilbert series of $I$ and that of $F:=B \otimes\left(L \oplus L^{\prime} x_{n} \oplus L^{\prime} x_{n}^{2} \oplus \cdots\right)$. Write $W=L \oplus L^{\prime} x_{n} \oplus L^{\prime} x_{n}^{2} \oplus \cdots$. Clearly $H_{F}(t)=H_{B}(t) \cdot H_{W}(t)$. We have

$$
H_{W}(t)=(n-1) t+(n-2) t^{2}+(n-2) t^{3}+\cdots=\left((n-1) t-t^{2}\right)(1-t)^{-1} .
$$

The exact sequence $0 \rightarrow I \rightarrow B \rightarrow B / I \rightarrow 0$ implies $H_{I}(t)=H_{B}(t)-H_{B / I}(t)$. Since $B$ is Koszul of global dimension 3, it follows that

$$
H_{B}(t)=\left(1-(n+1) t+(n+1) t^{2}-t^{3}\right)^{-1}
$$

by [Sm1, Theorem 5.9] and the isomorphism (4) of the previous section. By (i), $H_{B / I}(t)=(1-t)^{-2}$. Hence

$$
\begin{aligned}
H_{I}(t) & =\left(1-(n+1) t+(n+1) t^{2}-t^{3}\right)^{-1}-(1-t)^{-2} \\
& =\left(1-(n+1) t+(n+1) t^{2}-t^{3}\right)^{-1} \cdot\left((n-1) t-t^{2}\right)(1-t)^{-1} \\
& =H_{B}(t) \cdot H_{W}(t) \\
& =H_{F}(t) .
\end{aligned}
$$

Therefore $\mu$ is injective. So, (ii) follows.

Proof of the statement (iii) of Theorem 0.1 . If $n=2$, then $A=\mathbb{k}\left[x_{1}, x_{2}\right]$. We obtain that $B=A[z ; \bar{\delta}]$ is Noetherian, and hence coherent. Now assume $n \geq 4$. We only prove the statement when $j=n$ in the assumption, that is, $k_{n n}^{i}=0$ for all $i=1, \ldots, n$. When $j \neq n$, the statement can be proved similarly. By Lemma 2.2, there is an exact sequence $0 \longrightarrow I \longrightarrow B \longrightarrow B / I \longrightarrow 0$ such that $B / I$ is a polynomial algebra in two variables and $I$ is a free graded left $B$-module. By Pi , Proposition 3.2], $B$ is graded right coherent. Note that the left version of Lemma 2.2 (ii) holds too. Hence $B$ is also graded left coherent.

As a special case of the statement (iii) of Theorem 0.1, we have the following result, which can be viewed as a noncommutative version of GV, Theorem 4.3]. 
Proposition 2.4. Let $A$ be a connected graded 2-CY algebra. Then $A[z]$ is a graded coherent algebra.

Proof. By [Z1, Theorem 0.1] (also, cf. [Be, Proposition 3.4]), $A$ is defined by an invertible anti-symmetric matrix $M$, that is, $A=T(V) /\langle r\rangle$ with $r=\left(x_{1}, \ldots, x_{n}\right) \times$ $M\left(x_{1}, \ldots, x_{n}\right)^{t}$. For an invertible anti-symmetric matrix $M$, there is an invertible matrix $P$ such that $P^{t} M P$ is a standard invertible anti-symmetric matrix. Then the algebras defined by $M$ and $P^{t} M P$ respectively are isomorphic to each other. Hence we may assume that the anti-symmetric matrix $M$ itself is standard. Now by (iii) of Theorem 0.1 , we see that $A[z]$ is graded coherent.

Now assume that $B=A[z ; \bar{\delta}]$ is graded coherent. We may form a noncommutative projective space from $B$. Following $[\mathrm{Po}$, we denote by $\operatorname{coh} B$ the category of all finitely presented graded left $B$-modules, and by $\operatorname{fdim} B$ the category of all finite dimensional graded left $B$-modules. Since $B$ is graded coherent, $f \operatorname{dim} B$ is a Serre subcategory of $\operatorname{coh} B$. Hence the quotient category

$$
\operatorname{cohproj} B:=\operatorname{coh} B / \operatorname{fdim} B
$$

is also an abelian category. Since $B$ is Koszul and 3-CY, $B$ is Artin-Schelter regular with Gorenstein parameter -3 . Hence the Beilinson algebra of $B$ (for the terminology, see [MM, Definition 4.7]) is

$$
\nabla B=\left(\begin{array}{ccc}
\mathbb{k} & B_{1} & B_{2} \\
0 & \mathbb{k} & B_{1} \\
0 & 0 & \mathbb{k}
\end{array}\right)
$$

Let $\bmod \nabla B$ be the category of finite dimensional left $\nabla B$-modules. Then by $\mathrm{MM}$, Theorem 4.14], we have the following corollary.

Corollary 2.5. If the conditions of Theorem 0.1 are satisfied, then there is an equivalence of triangulated categories:

$$
D^{b}(\operatorname{cohproj} B) \cong D^{b}(\bmod \nabla B),
$$

where $D^{b}(-)$ is the bounded derived category of the corresponding abelian category.

\section{ACKNOWLEDGEMENTS}

The authors are very grateful to the referee for the careful reading of the manuscript and the numerous comments and suggestions which have greatly improved the paper. In particular, Remark 0.2 was suggested to the authors by the referee. The work was supported by an FWO-grant and grants from NSFC (No. 11171067) and NSF of Zhejiang Province (No. LY12A01013). This work was also supported in part by SRF for ROCS, SEM.

\section{REFERENCES}

[Be] Roland Berger, Gerasimov's theorem and N-Koszul algebras, J. Lond. Math. Soc. (2) 79 (2009), no. 3, 631-648, DOI 10.1112/jlms/jdp005. MR2506690 (2010d:16034)

[Bo] Raf Bocklandt, Graded Calabi Yau algebras of dimension 3, J. Pure Appl. Algebra 212 (2008), no. 1, 14-32, DOI 10.1016/j.jpaa.2007.03.009. MR2355031 (2008h:16013)

[BSW] Raf Bocklandt, Travis Schedler, and Michael Wemyss, Superpotentials and higher order derivations, J. Pure Appl. Algebra 214 (2010), no. 9, 1501-1522, DOI 10.1016/j.jpaa.2009.07.013. MR.2593679 (2011e:16022)

[DV] Michel Dubois-Violette, Multilinear forms and graded algebras, J. Algebra 317 (2007), no. 1, 198-225, DOI 10.1016/j.jalgebra.2007.02.007. MR2360146(2010e:16011)

[Gin] V. Ginzburg, Calabi-Yau algebras, arXiv:math/0612139. 
[GS] N. S. Gopalakrishnan and R. Sridharan, Homological dimension of Ore-extensions, Pacific J. Math. 19 (1966), 67-75. MR0200324(34 \#223)

[GV] B. V. Greenberg and W. V. Vasconcelos, Coherence of polynomial rings, Proc. Amer. Math. Soc. 54 (1976), 59-64. MR0417164 (54 \#5223)

[HVZ1] Ji-Wei He, Fred Van Oystaeyen, and Yinhuo Zhang, Hopf algebra actions on differential graded algebras and applications, Bull. Belg. Math. Soc. Simon Stevin 18 (2011), no. 1, 99-111. MR2809906 (2012d:16031)

[HVZ2] Ji-Wei He, Fred Van Oystaeyen, and Yinhuo Zhang, Deformations of Koszul ArtinSchelter Gorenstein algebras, Manuscripta Math. 141 (2013), no. 3-4, 463-483, DOI 10.1007/s00229-012-0580-z. MR3062595

[MM] Hiroyuki Minamoto and Izuru Mori, The structure of AS-Gorenstein algebras, Adv. Math. 226 (2011), no. 5, 4061-4095, DOI 10.1016/j.aim.2010.11.004. MR2770441(2012h:16053)

[Ph] Christopher Phan, The Yoneda algebra of a graded Ore extension, Comm. Algebra 40 (2012), no. 3, 834-844, DOI 10.1080/00927872.2010.539584. MR2899911

[Pi] Dmitri Piontkovski, Coherent algebras and noncommutative projective lines, J. Algebra 319 (2008), no. 8, 3280-3290, DOI 10.1016/j.jalgebra.2007.07.010. MR2408318 (2009e:14004)

[Po] A. Polishchuk, Noncommutative proj and coherent algebras, Math. Res. Lett. 12 (2005), no. 1, 63-74. MR2122731 (2005k:14003)

[Sm1] S. Paul Smith, Some finite-dimensional algebras related to elliptic curves, Representation theory of algebras and related topics (Mexico City, 1994), CMS Conf. Proc., vol. 19, Amer. Math. Soc., Providence, RI, 1996, pp. 315-348. MR1388568(97e:16053)

[Sm2] S. P. Smith, A 3-Calabi-Yau algebra with $G_{2}$ symmetry constructed from the Octonions, arXiv:1104.3824v1.

[So] J. Soublin, Un anneau cohérent dont l'anneau des polynômes n'est pas cohérent, C. R. Acad. Sci. Paris Sér. A-B 267 (1968), A241-A243.

[Z1] James J. Zhang, Non-Noetherian regular rings of dimension 2, Proc. Amer. Math. Soc. 126 (1998), no. 6, 1645-1653, DOI 10.1090/S0002-9939-98-04480-3. MR1459158 (98g:16007)

[Z2] James J. Zhang, Quadratic algebras with few relations, Glasgow Math. J. 39 (1997), no. 3, 323-332, DOI 10.1017/S0017089500032249. MR1484574 (98i:16011)

Department of Mathematics, Shaoxing College of Arts and Sciences, Shaoxing Zhejiang 312000, People's Republic of China; And Department of Mathematics and ComPuter Science, University of Antwerp, Middelheimlaan 1, B-2020 Antwerp, Belgium

E-mail address: jwhe@usx.edu.cn

Department of Mathematics and Computer Science, University of Antwerp, MiddelHeimlaAn 1, B-2020 Antwerp, Belgium

E-mail address: fred.vanoystaeyen@ua.ac.be

Department WNi, University of Hasselt, Universitaire Campus, 3590 Diepenbeek, BELGIUM

E-mail address: yinhuo.zhang@uhasselt.be 